\title{
Optimization of surgical parameters based on patient-specific models: application to arcuate keratotomy
}

Oskar Truffer MSc ${ }^{1}$, Daniel Abler PhD ${ }^{1}$, Bojan Pajic MD ${ }^{2,3,4,5}$, Günther Grabner MD ${ }^{6}$, Hannes Kraker $\mathrm{MD}^{6}$, and Philippe Büchler $\mathrm{PhD}^{1}$

${ }^{1}$ Institute for Surgical Technology and Biomechanics, University of Bern, Stauffacherstrasse 78, CH 3014 Bern, Switzerland

${ }^{2}$ Eye Clinic Orasis, Swiss Eye Research Foundation, 5734 Reinach AG Switzerland

${ }^{3}$ Division of Ophthalmology, Department of Clinical Neurosciences, Geneva University Hospitals, Switzerland

${ }^{4}$ Department of Physics, Faculty of Sciences, University of Novi Sad, Trg Dositeja Obradovica 4, 21000 Novi Sad, Serbia

${ }^{5}$ Faculty of Medicine of the Military Medical academy, University of Defense, 11000 Belgrade, Serbia

${ }^{6}$ University Eye Clinic Salzburg, Paracelsus Medical University, Müllner Hauptstraße 48, 5020 Salzburg, Austria

Running head:

Optimization of Arcuate Keratotomy Incision Parameters

Contributor responsible for the manuscript and proofs:

Oskar Truffer

Institute for Surgical Technology and Biomechanics, University of Bern,

Stauffacherstrasse 78, CH- 3014 Bern, Switzerland

oskar.truffer@istb.unibe.ch

Disclaimer: This work was supported by the Swiss Innovation Promotion Agency (18790.1 PFLS-LS).

IRB: Ethikkommission für das Bundesland Salzburg, 415-E/1820/19-2015, 12.02.2015

Total word count: 3420 


\section{ABSTRACT}

Purpose - Determining surgical parameters for arcuate keratotomy by simulating the intervention with a patient specific model.

Setting - University Eye Clinic Salzburg, Paracelsus Medical University, Austria \& Institute for Surgical Technology and Biomechanics, University of Bern, Switzerland

Design - Computational modeling study.

Methods - We propose a new approach to plan arcuate keratotomy based on personalized finite element simulations. Based on this numerical tool, an optimization algorithm was implemented to determine the incision parameters that best met the surgeon's requirements while preserving the orientation of the astigmatism. Virtual surgeries were performed on a cohort of patients to compare the performance of our simulation-based approach to results based on Lindstrom and Donnenfeld nomograms, and to intrastromal interventions.

Results - Retrospective data on 28 patients showed that personalized simulation reproduces the surgically-induced change in astigmatism (Pearson correlation of 0.8 ). Patient specific simulation was used to examine strategies for arcuate interventions on 621 corneal topographies. Lindstrom nomograms resulted in low postsurgical astigmatism (0.03D \pm 0.3 ) but frequent overcorrections $(20 \%)$. Donnenfeld nomograms and intrastromal incisions showed a small amount of overcorrection (1.5\%), but a wider spread in astigmatism $(0.63 \mathrm{D} \pm 0.35 \mathrm{D}$ and $0.48 \mathrm{D} \pm 0.50 \mathrm{D}$, respectively). In contrast, our numerical parameter optimization approach led to postoperative astigmatism values $(0.40 \mathrm{D} \pm 0.08 \mathrm{D}, 0.20 \mathrm{D} \pm 0.08 \mathrm{D}$, 
Journal of Cataract and Refractive Surgery

$0.04 \mathrm{D} \pm 0.13 \mathrm{D}$ ) that closely matched the targeted astigmatism of $0.4 \mathrm{D}, 0.2 \mathrm{D}$, and 0.0

$D$, while keeping the number of over-corrections low $(<1.5 \%)$.

Conclusions - Using numerical modelling to optimize surgical parameters for arcuate keratotomy leads to more reliable postoperative astigmatism and limits the risks of overcorrection. 


\section{INTRODUCTION}

Over 24.5 million cataract surgeries were performed worldwide in 2016. Although new techniques such as sutureless clear corneal incision have been introduced to replace limbal incisions, cataract interventions frequently induce undesired astigmatism. A recent study reported an average induced astigmatism between $0.77 \mathrm{D}$ and $1.29 \mathrm{D}$ depending on whether the clear corneal incision was performed with a temporal or superior approach, confirming results from the late nineties of induced astigmatism between $0.62 \mathrm{D}$ and 1.44 D. ${ }^{1-2}$ About two-thirds of patients also have a pre-existing astigmatism over $1.0 \mathrm{D},{ }^{3-4}$ which also requires some correction to optimize postoperative visual acuity. Thus, cataract surgery has evolved towards refractive surgery that aims to restore spectacle-free vision for the patient.

Arcuate keratotomy (AK) corrects both the astigmatism induced by the cataract incision and pre-existing astigmatism. Small incisions are made in the steep meridians to flatten the cornea. The position, length, and depth of the incisions are guided by nomograms based on the patient's amount of astigmatism. However, several studies suggest that AK planning is difficult, and postsurgical astigmatism can remain high. Even the use of a femtosecond laser for AK results in an average postoperative astigmatism of up to $0.9 \mathrm{D}$, and in some cases over- or undercorrection. ${ }^{5-9}$ The major problem with the current approach is that existing nomograms are based on statistical data from large numbers of patients, which therefore cannot account for individual anatomical features such as corneal thickness, detailed topography, and higher order aberrations. Moreover, any 
overcorrection has to be avoided. Clinicians thus tend toward a more conservative approach when choosing parameters, which often leads to undercorrection.

Planning solutions based on mechanical simulations, typically performed using the finite element method, can predict the optical outcome induced by the planned surgical incisions, before performing the actual surgery. The objective of this study was to rely on these numerical predictions to develop an optimization algorithm able to predict optimal incision parameters for AK surgery on each individual patient. Our hypothesis is that this model-based optimization of the surgical parameters helps reduce the variability in postoperative astigmatism and the risk of overcorrection. In this study, we first characterized the performance of our simulation tool by comparing the numerical predictions to clinical data. Then, we developed an approach to optimize surgical parameter and evaluated our optimization approach by comparing its results to conventional AK planning, which is based on existing clinical nomograms. 


\section{MATERIALS \& METHODS}

This study consists of four main phases (Figure 1). The first phase consisted in the selection of the patient dataset (Figure 1A). In the second phase, the numerical AK simulation framework was validated in a retrospective observational study on patients treated with intrastromal incisions (Figure 1B). In the third phase, the simulation platform evaluated the outcome of the AK treatment on a cohort of patients; the parameters of the intervention were either based on clinical nomograms or optimized using the proposed simulation platform (Figure 1C). In the fourth phase, we analyzed the change in astigmatism resulting from the incision parameters chosen by both the nomogram and the optimization approaches, and deduced a multidimensional nomogram for the observed cases (Figure 1D).

\section{Patient Data}

The corneal topography of 700 patients was acquired using a Pentacam HR Scheimpflug camera (Oculus Optikgeräte $\mathrm{GmbH}$, Germany). This system scans corneal shape with an accuracy of a few microns. Patients with corneal pathologies or previous refractive interventions that would be incompatible with AK were excluded, as well as those suffering from pathologies directly affecting corneal biomechanics or intraocular pressure. The remaining 649 topographic scans, with topographical astigmatism of the anterior surface between $0.5 \mathrm{D}$ and $3.0 \mathrm{D}$, were divided into two groups: 28 patients were used to evaluate the numerical model, and 621 patients were used to compare different planning strategies.

The 28 patients (13 men, 15 women) used for outcome evaluation had an intrastromal arcuate keratotomy performed with a femtosecond laser (Catalys 
Precision Laser System, Abbott Medical Optics Inc., Santa Ana, USA). The average age of the patients was $75.5 \pm 11.2$ years, and their average preoperative astigmatism was $1.24 \mathrm{D} \pm 0.43 \mathrm{D}$. Topographic information was recorded preoperatively, and postoperatively after an average follow-up of 163 days (90 - 499 days).

The 621 patients used to evaluate surgical strategies had an average age of $58 \pm 20$ years and preoperative astigmatism of $1.26 \pm 0.55 \mathrm{D}$.

Data were collected anonymously and in compliance with the local ethics committee regulations.

\section{Simulation and Optimization of Arcuate Keratotomy}

A numerical planning tool (Optimeyes, Optimo Medical AG, Biel, Switzerland) was used to simulate the mechanical deformation induced in the cornea by refractive intervention. This tool is based on the finite element method, includes a mechanical description of the stromal microstructure, ${ }^{10}$ and has already been validated on patients who underwent cataract surgery and laser keyhole endokeratophakia. ${ }^{11,12}$ The patient-specific models were obtained by morphing a spherical template to the patient-specific shapes of anterior and posterior corneal surfaces measured by a topographic device. ${ }^{11}$ The template model was built in such a way that it already included the incisions defining the given set of AK parameters including incision depth, position, length, and the number of incisions (Figure 2). The corneal topographies used to generate the numerical model represent the form of the eye stressed by the intraocular pressure. Therefore, an iterative algorithm was used to determine the stress free configuration of the patient-specific models under a physiological intraocular pressure of $15 \mathrm{mmHg}^{13}$. 
Because this planning tool enables us to predict the clinical outcome of any combination of cutting parameters, we could design an algorithm able to automatically change the surgical parameters until the optimal AK intervention is identified. We developed an iterative optimization method based on the LevenbergMarquardt algorithm ${ }^{14,15}$ to determine the cutting parameters required to reach a predefined level of astigmatism. The target for the optimization algorithm is to control the magnitude of the postoperative astigmatism, while preserving its preexisting orientation. In this study, the incisions were always performed on the steep axis of the cornea. Unlike the Lindstrom or Donnenfeld nomograms, where the number of incisions depends on the level of astigmatism, the optimization algorithm and intrastromal interventions always relied on two AK incisions. The initial values and the range of surgically admissible variations for each parameter are given in Table 1.

\section{Validation}

The evaluation dataset of 28 patients was used to assess the accuracy of the predictions achieved by the numerical model. These patients were treated with intrastromal arcuate incisions starting at $20 \%$ of the local pachymetry beneath the anterior corneal surface and ending at a depth of $80 \%$. All incisions were symmetrically placed within an optical zone of $7.0 \mathrm{~mm}$ and centered on the pupil. The incisions were cut with a side-cut angle of $115^{\circ}$ to be as parallel to the optical axis as feasible. Eyes with a preoperative cylinder of 1.0D or less were treated with $45^{\circ}$ arc-length incisions, while $60^{\circ}$ incisions were used for larger corrections (Figure 3). For each patient, the intervention was simulated with the planning tool described 
above, and the postsurgical outcome predicted by the model was compared to the actual postoperative shape of the patient's cornea. It is important to note that the numerical model only simulated the AK intervention, the effect of the Phaco incision for performing the cataract surgery was estimated by the surgeon.

\section{Population Simulations}

We performed virtual AK interventions based on different planning strategies on 621 patients of the evaluation group. Initially, the surgical parameters were based on the Lindstrom and Donnenfeld nomograms, and the intrastromal nomogram (Figure 3). In this study, we used the adaptation of the Donnenfeld nomogram proposed by Baharozian et al. ${ }^{16}$, which uses a shortened arcuate length to correct with-the-rule (WTR) astigmatism. This study provided detailed clinical outcome that can be used for direct comparison with the results of the numerical predictions ${ }^{16}$.

A second set of simulation was performed based on personalized optimization of the surgical parameters using our optimization algorithm described previously. This approach allows clinicians to define the desired target astigmatism for each patient. Here, we chose 3 levels of target astigmatism; $0.4 \mathrm{D}, 0.2 \mathrm{D}$, and 0.0 D. For each select target astigmatism, the optimization algorithm was evaluated on the complete database of patients.

Finally, the results obtained with the different planning strategies, nomograms and patient-specific optimization, were compared to each other. The complete prediction pipeline was fully automated; from model generation and execution of the prediction, to the calculation of the postoperative parameters.

\section{Evidence-based Nomogram}


Simulations of multiple AK incisions, corresponding to the 3 simulated nomograms and the 3 optimization targets, were performed for each of the 621 patients. The astigmatism change induced by all these interventions can be used to better understand the effect of the cutting parameters. Therefore, a linear regression was performed on these data to estimate the reduction in astigmatism as a function of the depth, position, and length of the incisions. This regression represents a multivariate nomogram for the observed cases, which was compared to the Lindstrom and Donnenfeld nomograms.

\section{Data Analysis}

We evaluated the accuracy of the simulation tool for AK surgery by comparing residual astigmatism and central curvature as predicted by the numerical model to the clinical results of 28 patients. Pearson correlation was assessed to analyze the effects of age, intraocular pressure, central corneal thickness, central corneal curvature, and gender on the surgically induced change in astigmatism.

We compared the surgical outcome obtained via the nomogram calculations among the 621 patients with the outcome achieved by our optimization algorithm. We also compared the magnitude of the final astigmatism and its orientation for each patient. Student's t-test was used to compare the postoperative astigmatism results obtained by the different approaches. The significance level was set to 0.05 .

We quantified the amount of overcorrection associated with each approach. Overcorrection was defined as an intervention in which the steep axis of astigmatism rotates more than $45^{\circ}$. For astigmatism below $0.25 \mathrm{D}$ the change in angle is not clinically relevant, therefore no overcorrection was considered below this threshold. 


\section{RESULTS}

\section{Validation}

The 28 patients used to evaluate the model accuracy had a preoperative astigmatism of $1.24 \mathrm{D} \pm 0.43 \mathrm{D}$, which was treated by intrastromal femtosecond laser AK as part of cataract surgery. The postoperative astigmatism of these eyes was $0.80 \mathrm{D} \pm 0.50 \mathrm{D}$, suggesting systematic undercorrection. No significant differences were observed between female and male patients $(p=0.801)$, or between left and right eyes $(p=0.683)$.

Finite element calculations were used to simulate the intrastromal AK of these 28 eyes. Residual astigmatism as predicted by the simulation, $0.70 \mathrm{D} \pm 0.39 \mathrm{D}$, was close to what was observed clinically: $0.80 \mathrm{D} \pm 0.50 \mathrm{D}$ (Figure 4). No statistical difference was observed between the clinical and predicted residual astigmatism $(p=0.118)$, and the correlation between clinical and predicted astigmatism was high (Pearson's $r=0.81$ ). The root mean square prediction error was 0.31D. For 93\% (26 out of 28 ) of the cases, the prediction error was below $0.5 \mathrm{D}$, and for more than $60 \%$ (17 out of 28 ) of the cases below $0.25 \mathrm{D}$ (Figure 3 ). However, for two data points the difference between clinical and predicted postoperative astigmatism was above $0.5 \mathrm{D}$, and in four cases the simulation predicted the axis would keep its orientation while a flipped axis was observed clinically.

\section{Population Simulations}

Average preoperative astigmatism of the 621 patients, $1.26 \mathrm{D} \pm 0.55 \mathrm{D}$, was significantly reduced by all AK interventions $(p<0.001)$. The approach proposed by Baharozian et al. proved to be conservative and resulted in a postoperative 
astigmatism of $0.63 \mathrm{D} \pm 0.35 \mathrm{D}$. This value is in line with the clinical data of $0.61 \mathrm{D} \pm 0.42 \mathrm{D}$ (Figure 5). ${ }^{16}$ This conservative approach successfully avoids overcorrection (1.5\% of the cases), but results in a relatively large postoperative astigmatism and a large standard deviation (Figure 6, a). This is in contrast to the Lindstrom nomogram, which resulted in an astigmatism close to zero $(0.03 D \pm 0.29 D)$, but at the cost of overcorrection in $20 \%$ of the cases (Figure $6, b)$. The intrastromal nomogram used in the evaluation step leads to postoperative astigmatism similar to the Donnenfeld nomogram $(0.48 \mathrm{D} \pm 0.50 \mathrm{D})$, but with less axis rotation (Figure $6, \mathrm{c}$ ). Clinically, the intrastromal approach is only used for an astigmatism below 1.5D; restricting the simulation to include only these patients results in a low postoperative astigmatism $(0.22 \mathrm{D} \pm 0.22 \mathrm{D})$ with an overcorrection rate of $1.5 \%$.

The proposed optimization algorithm reached the desired target astigmatism of $0.4 \mathrm{D}(0.40 \mathrm{D} \pm 0.08 \mathrm{D})$ with low overcorrection $(<0.5 \%)$ (Figure $6, d)$. The optimization algorithm's standard deviation (<0.01D) was smaller than that of both Lindstrom and Donnenfeld nomograms (0.3D). For the target astigmatism of 0.2D and $0.0 \mathrm{D}$, the optimization reached similar precision with a postoperative astigmatism of respectively $0.20 \mathrm{D} \pm 0.08 \mathrm{D}$ and $0.04 \mathrm{D} \pm 0.13 \mathrm{D}$ ((Figure 6 , e-f).

An overview of the accuracy of different planning strategies can be found in Figure 5. Notice that the postoperative astigmatism after planning with Baharozian's adaption of the Donnenfeld nomogram is reported once as seen in clinics and once as seen by our simulations. 


\section{Evidence-based Nomogram}

The large number of simulations $(>1800)$ carried out for this study enables us to identify the statistical relation between different cutting parameters and induced astigmatism. A linear regression was performed on the change of astigmatism $(\Delta A)$ obtained from all AK simulations, which describes the equivalent of a multivariate nomogram. Both state-of-the-art nomograms and the following multivariate nomogram obtained by a linear regression allows the surgeon to deduce the required incision parameters based on the preoperative astigmatism. But while for most nomograms only the arc length can be adjusted, using the multivariate nomogram all parameters can be adjusted;

$$
\Delta A=-0.54+1.34 d^{\prime}-0.63 p^{\prime}+2.74 l^{\prime}
$$

where $d^{\prime}, p^{\prime}$, and $I^{\prime}$ are respectively the normalized incision depth, half optical zone, and arcuate length. These parameters ranged from zero to one and were normalized according to the maximal and minimal values reported in Table 1. For example, the normalized length for an arcuate length of $30^{\circ}$ was obtained as,

$$
l^{\prime}=\frac{l-l_{\min }}{l_{\max }-l_{\min }}=\frac{30^{\circ}-20^{\circ}}{75^{\circ}-20^{\circ}}=0.18
$$

The fit of the linear model described $84 \%$ of the variability in the change of astigmatism $\left(R^{2}=0.84\right)$. The parameters of this model also indicate that the arcuate length has the largest effect on the induced astigmatism, followed by the depth of the incision. As expected the sign of the coefficient affecting the half optical zone is negative. This means that increasing the distance of the incision from the center of the cornea decreases the induced astigmatism. This parameter also has the lowest effect on the induced correction. 
When compared to the same set of surgical parameters, the induced astigmatism predicted by Lindstrom and Baharozian's adaptions of the Donnenfeld nomogram are close to the prediction of the optimized multivariate nomogram (Table 2). The Lindstrom nomogram underestimates the change induced by the intervention, especially for large correction factors (arc length $>37^{\circ}$ ). On the other hand, Donnenfeld mostly underestimates the induced change, but still induces overcorrection for the largest incision (arc length of $60^{\circ}$ ). Furthermore, the multivariate nomogram prediction lies between the two clinical nomograms, which indicates that even a multivariate nomogram will not significantly improve the clinical outcomes. This observation is confirmed by the large variability (up to $0.9 \mathrm{D}$ ) of the change in astigmatism calculated for different patients operated with identical sets of incision parameters (Table 2).

In the multivariate nomogram, the change in astigmatism induced by AK is 0.05D per degree of arcuate length. Wang et al. obtained comparable results by performing a regression on clinical data, reporting an induced change of astigmatism between $0.045 \mathrm{D}$ and $0.066 \mathrm{D}$ per degree. ${ }^{17}$ 


\section{DISCUSSION}

Our optimization approach based on personalized mechanical simulation provided more reliable incision parameters compared to conventional nomograms. These predicted incision parameters better corrected presurgical astigmatism to a predefined level while, at the same time, preventing overcorrection. The proposed optimization framework proved to be able to systematically reach the predefined target astigmatism with a low standard deviation $(<0.13 \mathrm{D})$ across the studied cohort. Our findings also suggest that the Lindstrom nomogram causes major overcorrection associated with large rotation of the steep astigmatism axis, while the parameters proposed by Baharozian, based on the Donnenfeld nomogram do not provide sufficient correction.

These results inform a new approach to optimizing the parameters of AK using patient-specific simulations of the intervention. Modern topographic devices provide patient specific maps of the cornea with a precision of a few microns, and femtosecond lasers allow refractive surgeons to execute preoperative AK planning accurately. The precision of these tools stands in contrast to the limited and often subjective information available to clinicians planning such interventions.

Indeed, nomograms based on population statistics cannot accurately predict change in astigmatism for a specific patient because they do not adequately account for individual patient anatomy and biomechanics. Using our large number of simulated interventions, we identified the statistical relation between incision parameters and change in astigmatism in a linear regression model. Unlike traditional clinical nomograms, this regression model includes the effect of all the cutting parameters and is not limited to accounting solely for the variation of arc 
length, keeping the depth and position of the cut constant. The resulting multivariate linear model explains $85 \%$ of the variability present in the datasets, which implies that the remaining $15 \%$ cannot be explained by the incision parameters but are related to patient-specific corneal parameters. This remaining variability has a rather large impact on the clinical outcomes, with postoperative astigmatism errors reaching up to one diopter. This result also indicates that, even with an optimized nomogram that takes all the incision parameters into account, accurate predictions of the postoperative astigmatism are not possible. The predictions of current nomograms are already close to the optimal regression model that we proposed based on population data, which means that no further improvements of the clinical outcome can be expected with this approach. Future methods have to rely on more personalized data, such as the one proposed in this work.

Predictions of the numerical model are corroborated by clinical data from Baharozian et al., describing post-surgical results following AK clinically. The achieved correction was accurately reproduced by the numerical model. Furthermore our study also confirms their findings concerning the position of the incisions: a higher amount of overcorrection is associated with the larger incision suggested for against-the-rule astigmatism. ${ }^{16}$ At the same time, we confirm the finding that slightly longer incisions for oblique astigmatism than with-the-rule astigmatism lead to near target correction of preoperative astigmatism.

One of the major limitations of this study results from its numerical model, which does not incorporate the complete variability characterizing the ocular system. More specifically, no method is currently available to determine the 
mechanical properties of the cornea in-vivo, despite recent technical developments such as Brillouin scattering ${ }^{18-20}$ or non-contact tonometry ${ }^{21,22}$. Consequently, while the corneal topography can be accurately characterized on each patient, the same material description was considered for all the patients. However, it has been suggested that patient-specific material properties play a secondary role when predicting mechanical responses ${ }^{23}$. In addition, the mechanical framework used in this study was previously validated for other refractive interventions, ${ }^{12,24}$ and we found no statistical differences between the prediction of the model and the residual astigmatism of patients treated with intrastromal AK. The high correlation between the numerical model and the surgical outcome, and the fact that we obtained corrections similar to those reported by Baharozian et al. ${ }^{16}$ provide rationales to trust the model predictions. Furthermore, the simulated intervention with our patients resulted in a correction per degree of arcuate length similar to that reported by Wang et al., ${ }^{17}$ which reinforces confidence in the results of the optimized nomogram with clinical data.

Computational studies using accurate and robust numerical models offer multiple key benefits over clinical investigations. They allow direct comparison of multiple surgical procedures with different parameters on the same patient. In addition, they enable 'experimental' AK studies using combinations of surgical parameters that would not be suitable to treat actual patients. Finally, the results based on such simulations provide a proof of concept for the optimization methods and therefore justify larger clinical trials. The validation of the prediction of the simulation platform based on observational studies showed that the model was able to reproduce clinical observations, which provides a degree of confidence necessary 
to initiate clinical investigations. Thorough clinical validation of this planning tool is required in order to provide sufficient evidences of the safety and reliability of the numerical predictions within a clinical environment.

While nomograms can support the surgeon by suggesting cutting parameters, they frequently result in under- or overcorrection. The proposed personalized prediction approach, on the other hand, enables surgeons to specify and accurately reach a target postsurgical astigmatism while increasing the safety of the procedure by avoiding overcorrections. Since it relies on a numerical model, this optimization technique additionally supplies an estimation of the resulting topography that enables the surgeon to correct the proposed surgical parameters if necessary.

\section{What was known}

- Planning and executing AK surgery with nomograms significantly reduces astigmatism. Under- and overcorrection are observed.

- Mechanical simulations can predict the outcome of cataract surgery and laser keyhole endokeratophakia.

\section{What this paper adds}

- About $15 \%$ of variability in the postsurgical astigmatism cannot be explained by a nomogram (up to $1 D$ ), but are related to the specific shape of each patient's cornea. 
Journal of Cataract and Refractive Surgery

- Patient-specific numerical models of AK surgery allow reaching a predefined astigmatism more accurately than existing nomograms, while increasing the safety of the procedure by avoiding overcorrections.

\section{ACKNOWLEDGEMENTS}

This work was supported by the Swiss Innovation Promotion Agency (18790.1 PFLSLS). 


\section{REFERENCES}

1. Simsek S, Yasar T, Demirok A, Cinal A, Yilmaz OF. Effect of superior and temporal clear corneal incisions on astigmatism after sutureless phacoemulsification. J Cataract Refract Surg. 1998;24(4):515-518. doi:10.1016/S0886-3350(98)80294-0

2. Nikose AS, Saha D, Laddha PM, Patil M. Surgically induced astigmatism after phacoemulsification by temporal clear corneal and superior clear corneal approach: a comparison. Clin Ophthalmol. 2018;12:65-70.

doi:10.2147/OPTH.S149709

3. Hoffmann PC, Hütz WW. Analysis of biometry and prevalence data for corneal astigmatism in 23239 eyes. J Cataract Refract Surg. 2010;36(9):1479-1485. doi:10.1016/j.jcrs.2010.02.025

4. Curragh DS, Hassett P. Prevalence of corneal astigmatism in an NHS cataract surgery practice in Northern Ireland. Ulster Med J. 2017;86(1):25-27.

5. Nejima R, Terada Y, Mori Y, Ogata M, Minami K, Miyata K. Clinical utility of femtosecond laser-assisted astigmatic keratotomy after cataract surgery. Jpn J Ophthalmol. 2015;59(4):209-215. doi:10.1007/s10384-015-0383-3

6. Chan TCY, Ng ALK, Cheng GPM, Wang Z, Woo VCP, Jhanji V. Corneal Astigmatism and Aberrations After Combined Femtosecond-Assisted Phacoemulsification and Arcuate Keratotomy: Two-Year Results. Am J Ophthalmol. 2016;170(2):83-90. doi:10.1016/j.ajo.2016.07.022

7. Vickers LA, Gupta PK. Femtosecond laser-assisted keratotomy. Curr Opin Ophthalmol. 2016;27(4):277-284. doi:10.1097/ICU.0000000000000267

8. Rückl T, Dexl AK, Bachernegg A, et al. Femtosecond laser-assisted intrastromal 
arcuate keratotomy to reduce corneal astigmatism. J Cataract Refract Surg. 2013;39(4):528-538. doi:10.1016/j.jcrs.2012.10.043

9. Monaco G, Scialdone A. Long-term outcomes of limbal relaxing incisions during cataract surgery: Aberrometric analysis. Clin Ophthalmol. 2015;9:15811587. doi:10.2147/OPTH.S89024

10. Studer H, Larrea X, Riedwyl H, Büchler P. Biomechanical model of human cornea based on stromal microstructure. J Biomech. 2010;43(5):836-842. doi:10.1016/j.jbiomech.2009.11.021

11. Studer HP, Riedwyl H, Amstutz C a., Hanson JVM, Büchler P. Patient-specific finite-element simulation of the human cornea: A clinical validation study on cataract surgery. J Biomech. 2013;46(4):751-758. doi:10.1016/j.jbiomech.2012.11.018

12. Studer HP, Pradhan KR, Reinstein DZ, et al. Biomechanical Modeling of Femtosecond Laser Keyhole endokeratophakia Surgery. J Refract Surg. 2015. doi:10.3928/1081597X-20150623-07

13. Elsheikh A, Whitford C, Hamarashid R, Kassem W, Joda A, Büchler P. Stress free configuration of the human eye. Med Eng Phys. 2013;35(2):211-216. doi:10.1016/j.medengphy.2012.09.006

14. Marquardt DW. An Algorithm for Least-Squares Estimation of Nonlinear Parameters. J Soc Ind Appl Math. 1963;11(2):431-441. doi:10.1137/0111030

15. Levenberg K. A Method for the Solution of Certain Non-Linear Problems in Least. Q App/ Math. 1944;2(278):164-168. doi:10.1090/qam/10666

16. Baharozian $\mathrm{CJ}$, Song $\mathrm{C}$, Hatch KM, Talamo JH. A novel nomogram for the treatment of astigmatism with femtosecond-laser arcuate incisions at the 
time of cataract surgery. Clin Ophthalmol. 2017;11:1841-1848.

doi:10.2147/OPTH.S141255

17. Wang L, Zhang S, Zhang Z, et al. Femtosecond laser penetrating corneal relaxing incisions combined with cataract surgery. J Cataract Refract Surg. 2016;42(7):995-1002. doi:10.1016/j.jcrs.2016.04.020

18. Scarcelli G, Besner S, Pineda R, Kalout P, Yun SH. In vivo biomechanical mapping of normal and keratoconus corneas. JAMA Ophthalmol. 2015;133(4):480-482. doi:10.1001/jamaophthalmol.2014.5641

19. Scarcelli G, Besner S, Pineda R, Yun SH. Biomechanical characterization of keratoconus corneas ex vivo with brillouin microscopy. Investig Ophthalmol Vis Sci. 2014;55(7):4490-4495. doi:10.1167/iovs.14-14450

20. Scarcelli G, Kling S, Quijano E, Pineda R, Marcos S, Yun SH. Brillouin microscopy of collagen crosslinking: noncontact depth-dependent analysis of corneal elastic modulus. Invest Ophthalmol Vis Sci. 2013;54(2):1418-1425. doi:10.1167/iovs.12-11387

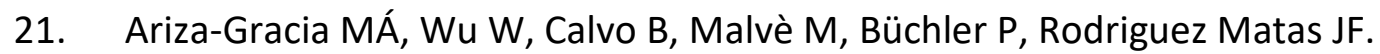
Fluid-structure simulation of a general non-contact tonometry. A required complexity? Comput Methods Appl Mech Eng. 2018;340:202-215. doi:10.1016/j.cma.2018.05.031

22. Sinha Roy A, Kurian M, Matalia H, Shetty R. Air-puff associated quantification of non-linear biomechanical properties of the human cornea in vivo. J Mech Behav Biomed Mater. 2015;48:173-182. doi:10.1016/j.jmbbm.2015.04.010

23. Ariza-Gracia M, Ortillés, Cristóbal J, Rodríguez Matas JF, Calvo B. A numericalexperimental protocol to characterize corneal tissue with an application to 
Journal of Cataract and Refractive Surgery

predict astigmatic keratotomy surgery. J Mech Behav Biomed Mater. 2017;74(June):304-314. doi:10.1016/j.jmbbm.2017.06.017

24. Studer HP, Riedwyl H, Amstutz CA, Hanson JVM, Büchler P. Patient-specific finite-element simulation of the human cornea: A clinical validation study on cataract surgery. J Biomech. 2013;46(4):751-758.

doi:10.1016/j.jbiomech.2012.11.018 


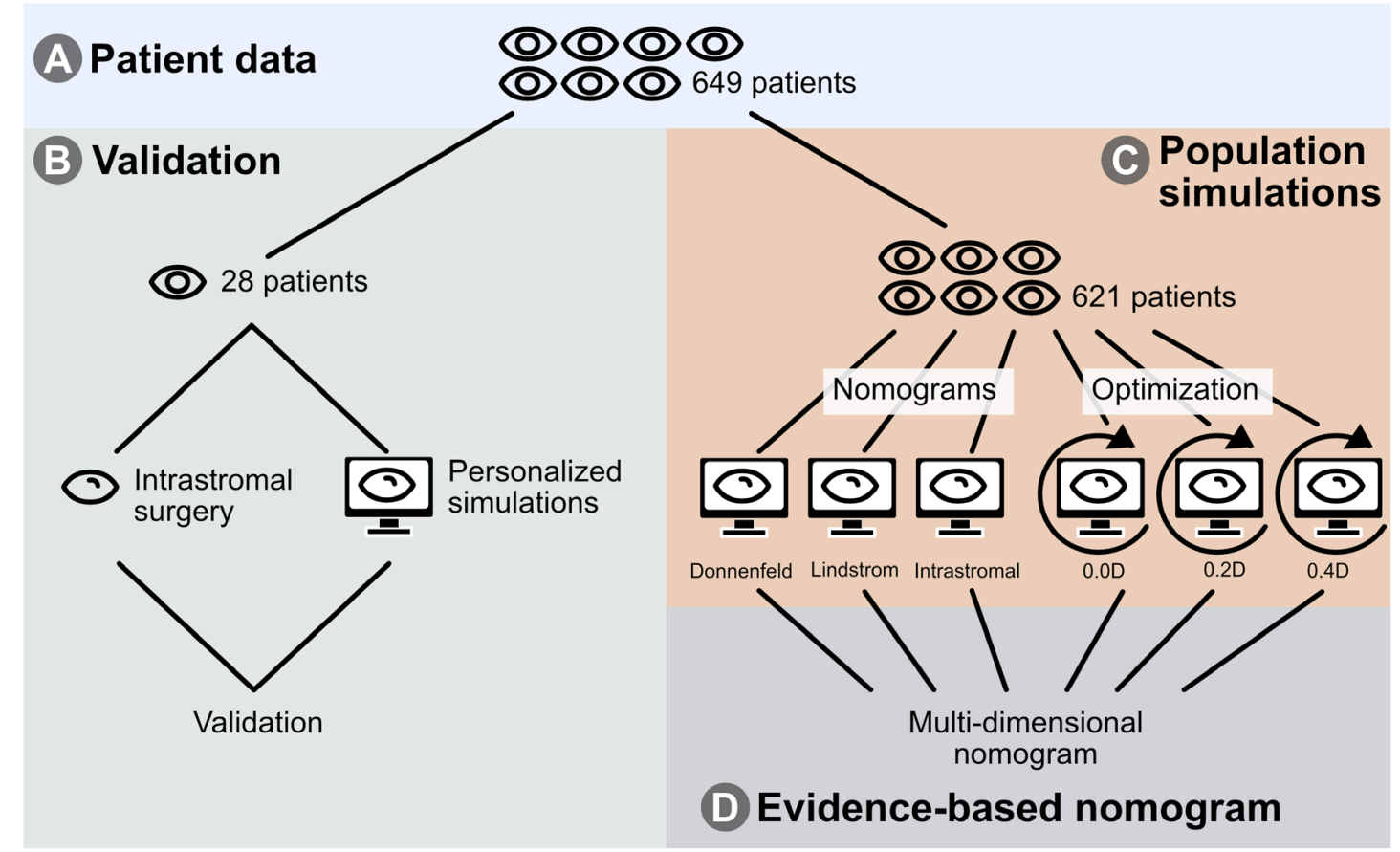

Figure 1 The paper covers several phases; Patient recruitment and data acquisition (A), validation of the patient-specific simulation framework (B), comparison of different planning strategies based on personalized mechanical simulations performed on a cohort of patients (C), and derivation of an optimal multidimensional nomogram based on all simulated surgeries. 


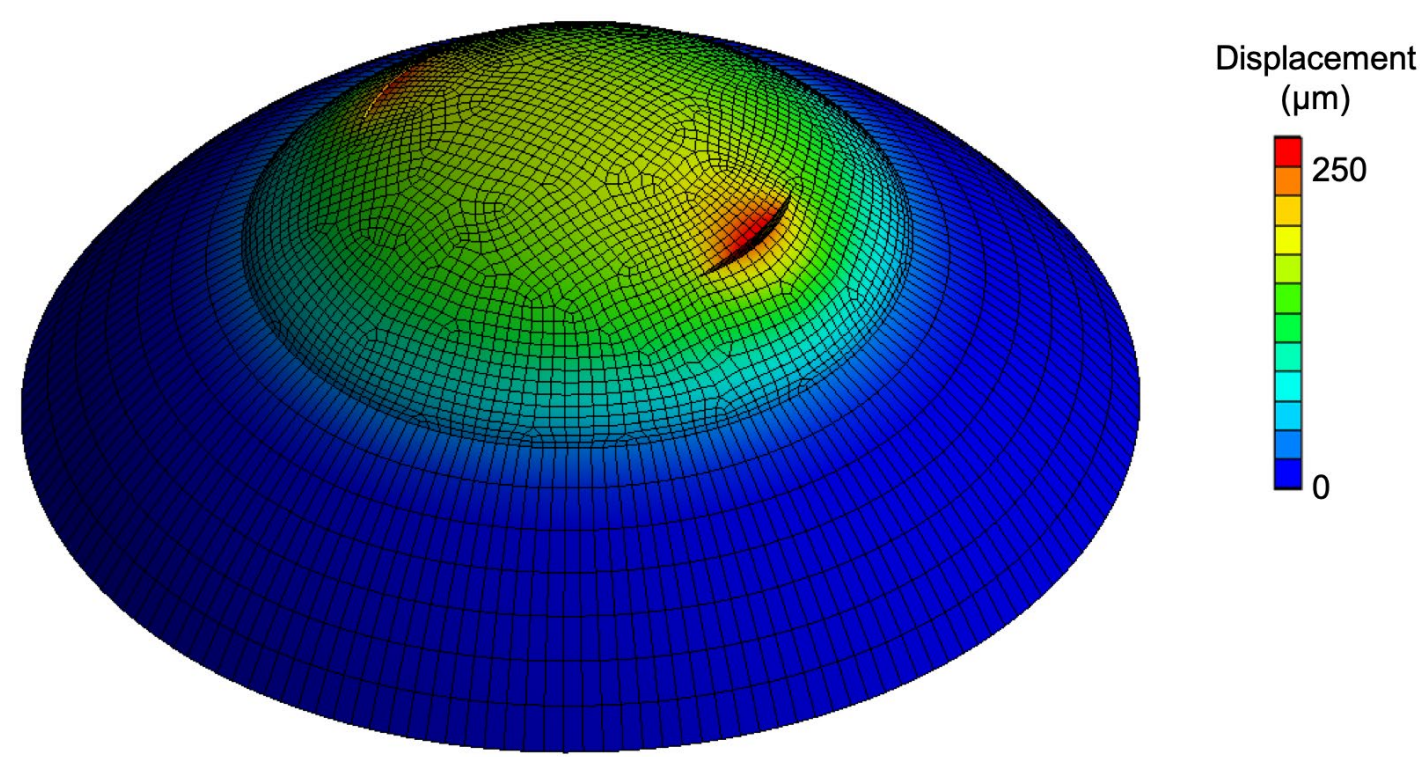

Figure 2 Finite element model showing the cornea and the sclera, as well as the positions of the AK incisions. The colors indicate the displacements induced in the cornea by the AK incisions. The modified corneal shape can be used to calculate the amplitude and orientation of the postsurgical astigmatism. 

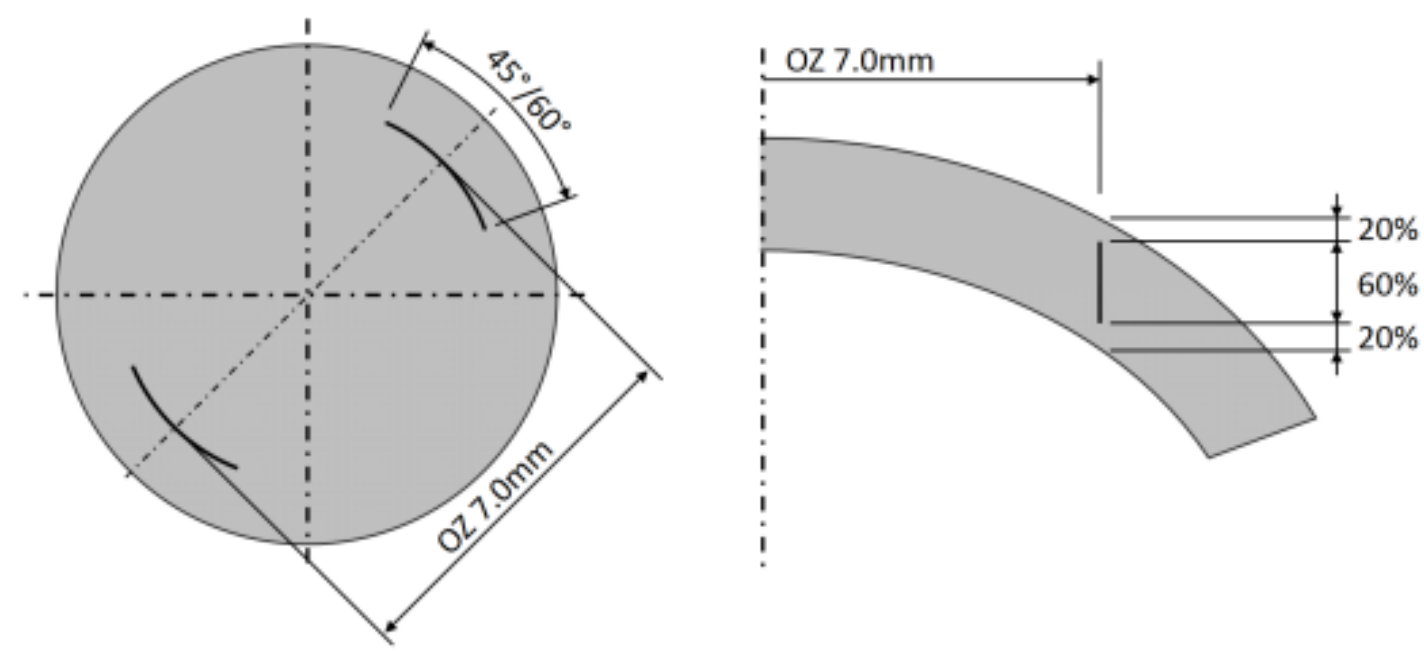

Figure 3 Schematic representation of the intrastromal incision: top view of the cornea (left) and cross section (right), showing the placement of the incision within the stroma. The incisions are positioned in the $7.0 \mathrm{~mm}$ optical zone with an arc-length of $45^{\circ}$ for pre-existing astigmatism lower than $1 \mathrm{D}$, or $60^{\circ}$ otherwise. The incisions were symmetric and centered on the pupil. The distance from the anterior surface to the beginning of the incision was $20 \%$ of local pachymetry in all cases. Similarly, the distance from the posterior surface to the end of the incision was also $20 \%$ of the local corneal thickness. 


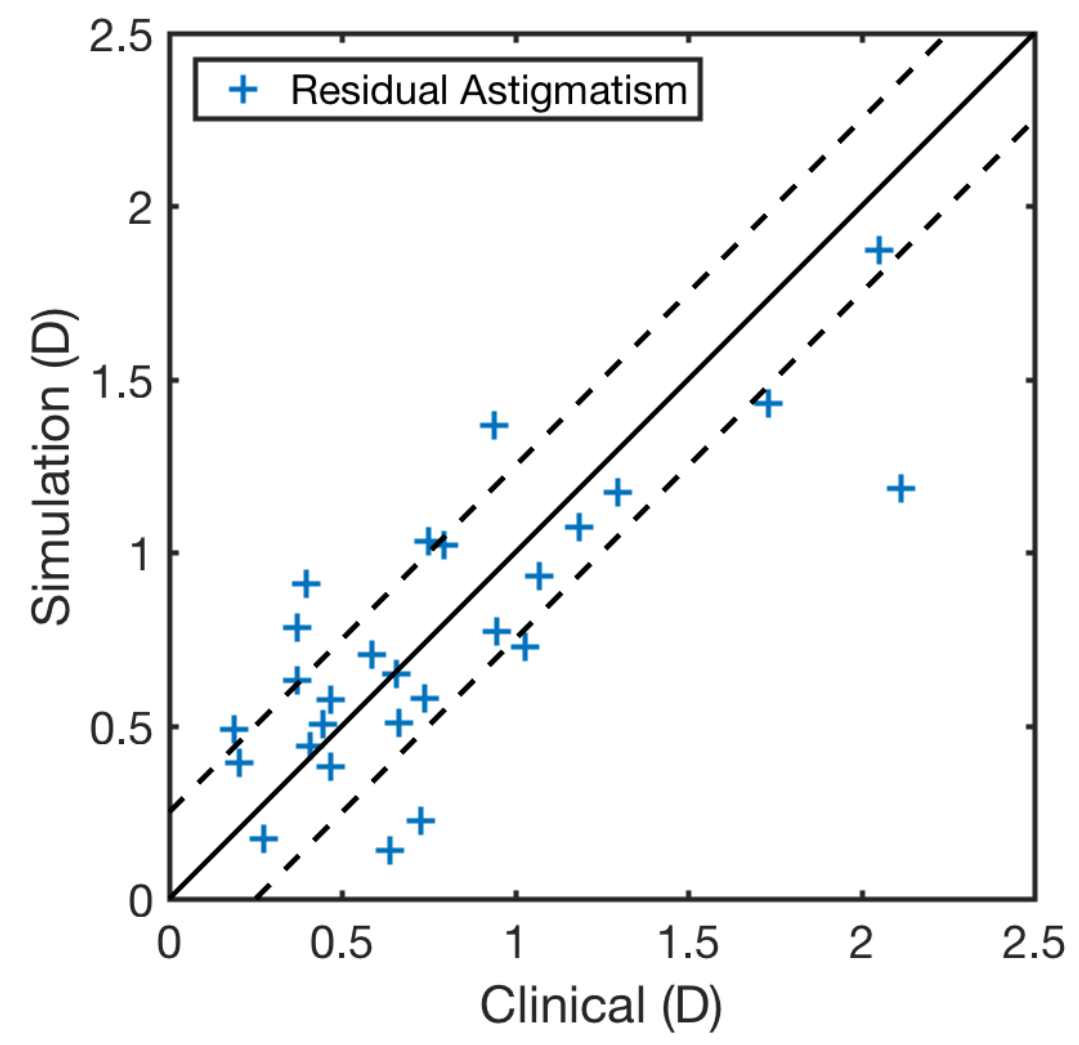

Figure 4 Predicted postoperative astigmatism compared to clinical postoperative astigmatism (residual astigmatism) indicates high predictive power of patient-specific arcuate keratotomy surgery simulation $(r=0.81)$. 


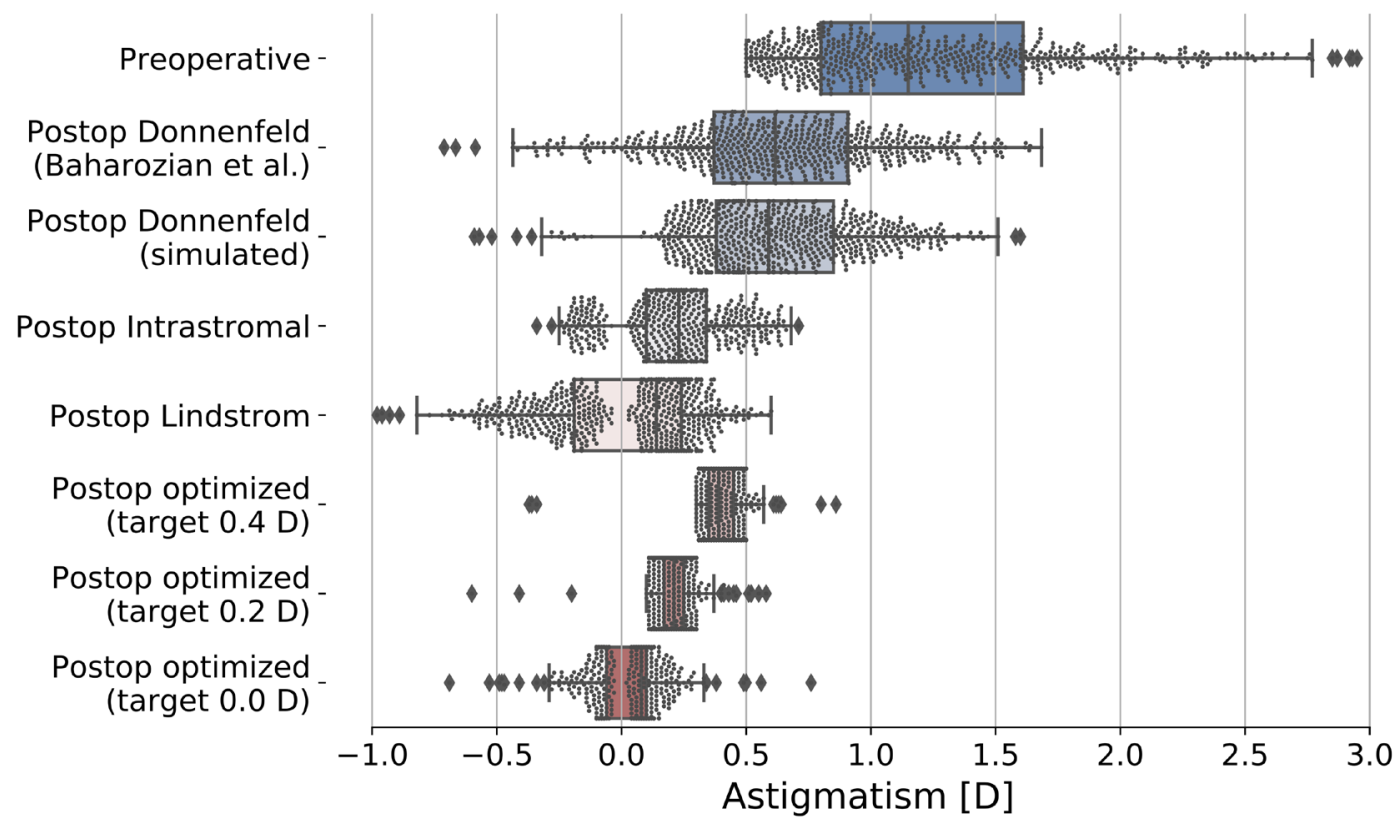

Figure 5 Pre- and postoperative astigmatism when using different approaches to plan the AK surgery. Interventions that change the astigmatism by more than $45^{\circ}$ are denoted as negative numbers. The optimized approach leads to lower variation in the outcome and can achieve the desired astigmatism reliably. The area in red depicts the overcorrected cases. Values very close to zero $(<0.05 \mathrm{D})$ are rarely seen as this would require a rotation symmetric cornea. 
(a) Donnenfeld

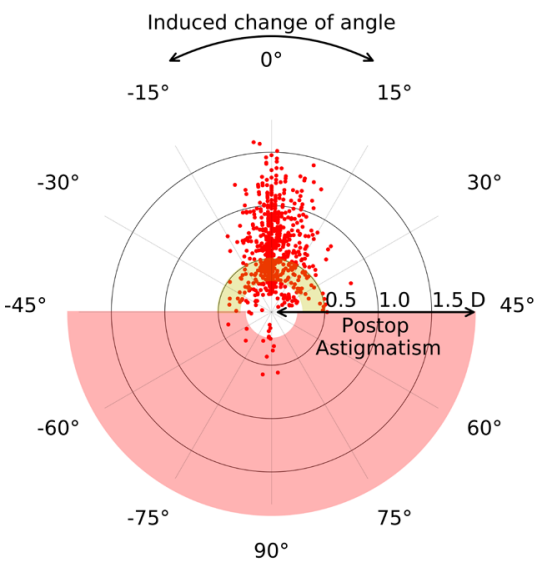

(b) Lindström

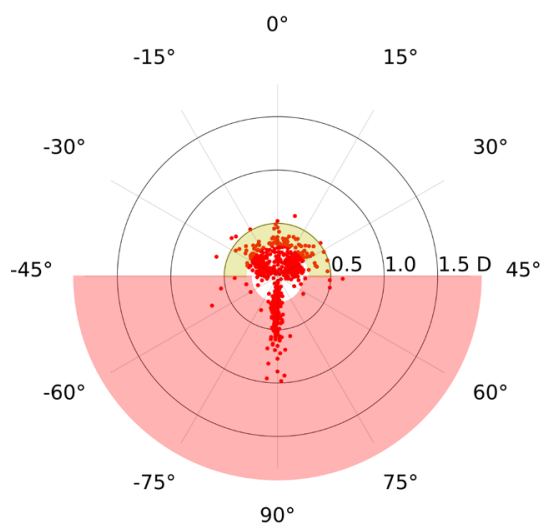

(c) Intrastromal

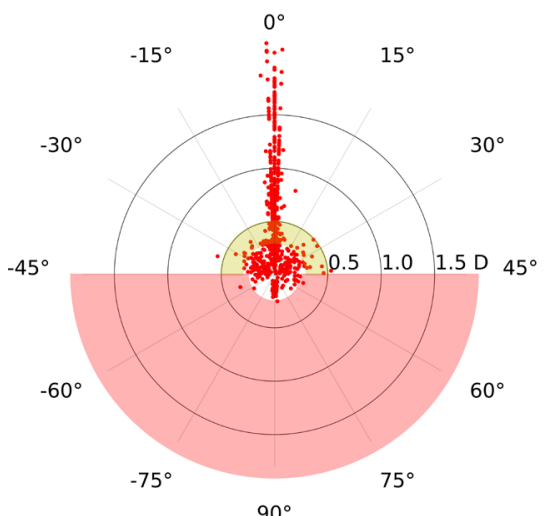

$90^{\circ}$

Overcorrection

(Change in angle $>45^{\circ}$ ) (d) Optimized - 0.4D

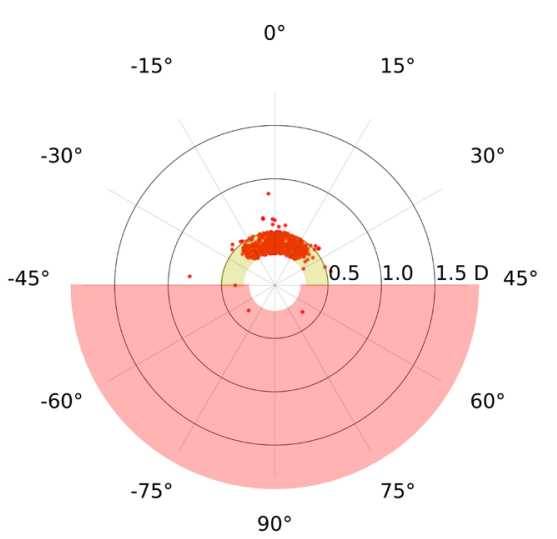

(e) Optimized - 0.2D

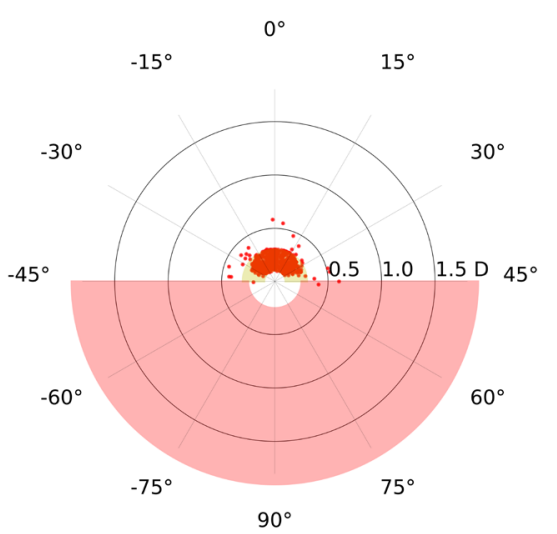

(e) Optimized - 0.2D

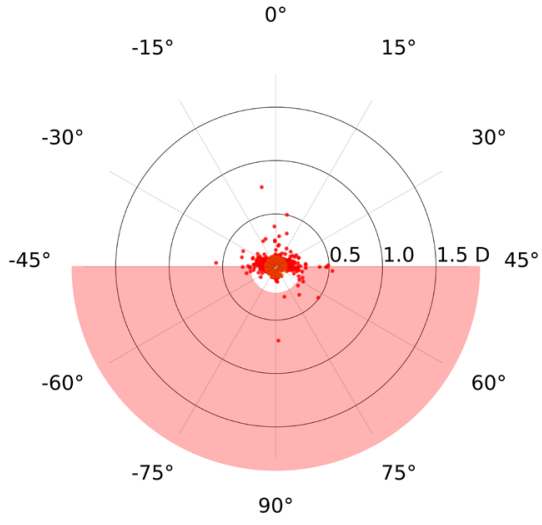

Desired result

(Astigmatism 0.3 D - 0.5 D)

Figure 6 The outcome of each AK simulation is shown using polar plots. The distance from the center represents the postoperative astigmatism while the polar position describes the rotation of the steep meridian induced by the AK surgery. No rotation of the steep axis corresponds to the angle of $0^{\circ}$ along the positive vertical axis, while axis rotation of more than $+45^{\circ}$ or less than $-45^{\circ}$ corresponds to overcorrection (red area). Each AK surgery is shown as a red dot on the diagram; the yellow region indicates the target astigmatism. The figure compares the outcome of the Donnenfeld (a), Lindstrom (b), and intrastromal (c) nomograms to the proposed optimization methods with different target astigmatism of $0.4 \mathrm{D}(\mathrm{d}), 0.2 \mathrm{D}(\mathrm{e})$ and $0.0 \mathrm{D}$ (f) based on 621 patients. 


\begin{tabular}{ccc}
\hline Parameter & Initial value & Value range \\
\hline Depth (\% corneal thickness) & $80 \%$ & $20 \%-90 \%$ \\
Arcuate length $\left(^{\circ}\right)$ & $30^{\circ}$ & $20^{\circ}-75^{\circ}$ \\
Distance from the center $(\mathrm{mm})$ & $4 \mathrm{~mm}$ & $3.5-5.5 \mathrm{~mm}$ \\
\hline
\end{tabular}

Table 1 Initial value and upper/lower limits of the AK parameters used by the optimization algorithm.

\begin{tabular}{ccccccc}
\hline \multicolumn{2}{c}{ Surgical Parameters } & Lindström & $\begin{array}{c}\text { Donnenfeld } \\
\text { (ATR) }\end{array}$ & $\begin{array}{c}\text { Multivariate } \\
\text { nomogram }\end{array}$ & $\begin{array}{c}\text { Population } \\
\text { simulation }\end{array}$ \\
\hline Arc length & Depth & $\begin{array}{c}\text { Optical } \\
\text { zone }\end{array}$ & & & & \\
\hline $30^{\circ}$ & $90 \%$ & $8 \mathrm{~mm}$ & $0.9 \mathrm{D}$ & - & $1.1 \mathrm{D}$ & $0.6 \mathrm{D}-1.25 \mathrm{D}$ \\
$45^{\circ}$ & $90 \%$ & $8 \mathrm{~mm}$ & $1.7 \mathrm{D}$ & - & $1.75 \mathrm{D}$ & $1.7 \mathrm{D}-2.5 \mathrm{D}$ \\
$60^{\circ}$ & $90 \%$ & $8 \mathrm{~mm}$ & $2.5 \mathrm{D}$ & - & $2.5 \mathrm{D}$ & $3.0 \mathrm{D}-3.9 \mathrm{D}$ \\
\hline $30^{\circ}$ & $90 \%$ & $9 \mathrm{~mm}$ & - & $0.75 \mathrm{D}$ & $0.75 \mathrm{D}$ & $0.5 \mathrm{D}-1.0 \mathrm{D}$ \\
$45^{\circ}$ & $90 \%$ & $9 \mathrm{~mm}$ & - & N/A & $1.5 \mathrm{D}$ & $0.9 \mathrm{D}-1.5 \mathrm{D}$ \\
$60^{\circ}$ & $90 \%$ & $9 \mathrm{~mm}$ & - & $1.5 \mathrm{D}$ & $2.2 \mathrm{D}$ & $1.5 \mathrm{D}-2.1 \mathrm{D}$ \\
\hline
\end{tabular}

Table 2 Change of astigmatism for different arc length predicted for the Linstörm, the Donnenfeld, and multivariate nomograms, on the cohort of 621 patients included in this study. These results are presented with an incision depth and optical corresponding respectively to the Linström and Donnenfeld nomogram. The Lindström nomgram underestimates the change of astigmatism, while the Donnenfeld nomogram provides a better estimate of the actual induced astigmatism, similar to the proposed multivariate nomogram. Nevertheless, the change in astigmatism observed in the population showed a large variability, which is not accounted for by any nomogram. 